Página inicial: 591 - Página final: 602

Tipo de artículo: de Reflexión derivada de investigación

\title{
Construcción de ciudadanías en sociedades con altos niveles de conflictividad y crisis a través de una iniciativa pedagógica
}

\author{
Construction of citizenships in societies with high conflict and crisis levels \\ by means of a pedagogical initiative.
}

Recibido: noviembre 2015 Revisado: abril 2016 Aceptado: mayo 2016

Por: Edgar Ramirez Monsalve* y Jovani Alberto Jiménez Builes**

\section{Resumen.}

Se propone un proceso donde lo constitucional cope un espacio de formación, en el entendido que éste, recoge aspectos centrales de orden ético, cultural y legal de la formación ciudadana, de otro lado, los referentes formativos se inscriben en una cultura democrática de respeto, acatamiento y fomento de los derechos humanos, la pluralidad y el tratamiento negociado de los conflictos. La iniciativa consigna aspectos relativos a la gestión del desarrollo, la formación de redes educadoras, estrategias públicas y de comunicación para la construcción del debate público como aspecto formador, orientadas al territorio colombiano.

\section{Palabras claves.}

Educación, pedagogía, democracia, ciudadanía, conflicto armado.

\begin{abstract}
.
A training process is proposed, where the constitutional deals with a training space, on the understanding that it refers to central aspects of ethical, cultural, and legal order of citizen formation, on the other hand, the educational benchmarks are enrolled in a democratic culture of respect, observance and promotion of human rights, plurality and negotiated treatment of conflicts. The initiative provides aspects relating to the management of development, the formation of educational networking, public and communication strategies for the construction of the public debate as a training aspect, geared toward the Colombian territory.
\end{abstract}

\section{Key words.}

Education, Pedagogy, Democracy, Citizenship, Armed Conflict.

\footnotetext{
* Doctor en Educación y Sociedad de la Universidad de Sevilla - España. Profesor Titular de la Universidad Nacional de Colombia e Integrante del Grupo de Investigación Inteligencia Artificial en Educación. eramirezmo@ unal.edu.co

** Doctor en Ingeniería Área Sistemas e Informática de la Universidad Nacional de Colombia. Profesor Titular de la Universidad Nacional de Colombia e Integrante del Grupo de Investigación Inteligencia Artificial en Educación. jajimen1@unal.edu.co
} 


\section{Introducción.}

La cultura ciudadana conlleva un proceso de aprendizaje que atraviesa los múltiples relacionamientos, conductas, comportamientos, ambientes, contextos y valores de los sujetos sociales (Ver Figura 1). La vida ciudadana se contextualiza en diferentes ámbitos y esferas del quehacer social, político, ético, cultural y ambiental, donde las transversalidades de lo cotidiano, lo espontáneo, lo institucional, lo estructural y lo direccionado presentan conjugaciones heterogéneas, plurales y problemáticas. La condición moderna de la ciudadanía se ejerce en espacios donde la otredad y la diferencia marcan rumbos impredecibles.

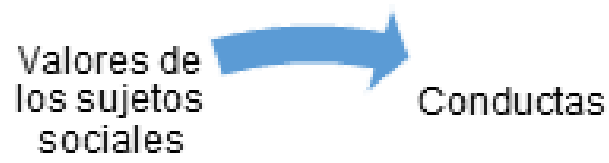

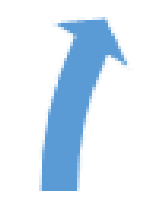

Contextos

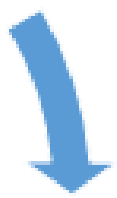

Comportamientos

Figura 1. Procesos de aprendizaje de la cultura ciudadana

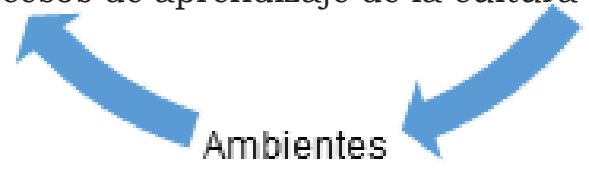

La cultura ciudadana como fenómeno socio-cultural y político no se agota en el ejercicio de los derechos y responsabilidades del sujeto-ciudadano. Es un proceso que inicia desde la socialización familiar, escolar, barrial, citadina y que toca las formas organizativas y participativas de la denominada sociedad civil, hacia el ejercicio político participativo (Vargas, 2001).

Entender que la condición personal y subjetiva presupone la existencia de un individuo bio-psico-social que incide con sus conductas, comportamientos y actitudes frente al medio donde interactúa, es concebir los procesos sociales sobre la base de procesos humanos que necesitan proyectarse de forma colectiva y direccionados para el logro de condiciones de bienestar y desarrollo local con visión global. 


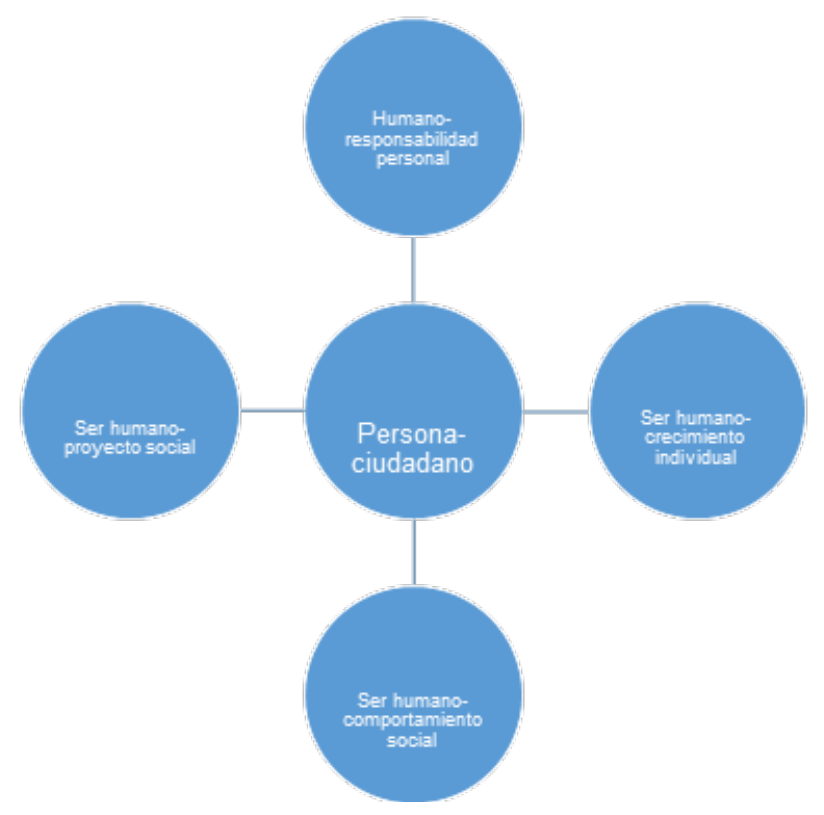

Figura 2. Condiciones de la persona-ciudadano

En la construcción de un sujeto social complejo y diverso, se deben incluir reflexiones personales y colectivas con aunamiento de responsabilidades y articulación de esfuerzos y recursos orientados a materializar propuestas de ciudadanía, que den sustento al desarrollo humano.

Dar a conocer a los ciudadanos, el marco legal, politico y societal que permita que todos participen de la vida política y pública como sujetos conscientes y activos para alcanzar la civilidad y el bienestar del territorio colombiano, es la base del proceso de formación para la cualificación del ejercicio de la ciudadanía (Botia, 1998).

Es necesario promover la cultura política que atraviesa las reglas del juego ciudadano y los conceptos políticos básicos de nuestro ordenamiento político-constitucional. Esto permite dar a conocer los deberes de la persona y el ciudadano, así como la importancia de la democracia y la participación ciudadana en el nuevo orden constitucional y legal, que tiene sus raíces en principios planificadores y de gestión social.

Este panorama formativo contribuye a fortalecer la convivencia ciudadana alrededor de la participación, planeación local del desarrollo, metodologías sociales para el tratamiento no violento de los conflictos, que forman parte de las dinámicas sociales que vinculan a las organizaciones a las diferentes expresiones de la sociedad civil y la administración pública (Álvarez, Dagnino, \& Escobar, 1998) (Ver Figura 3). 


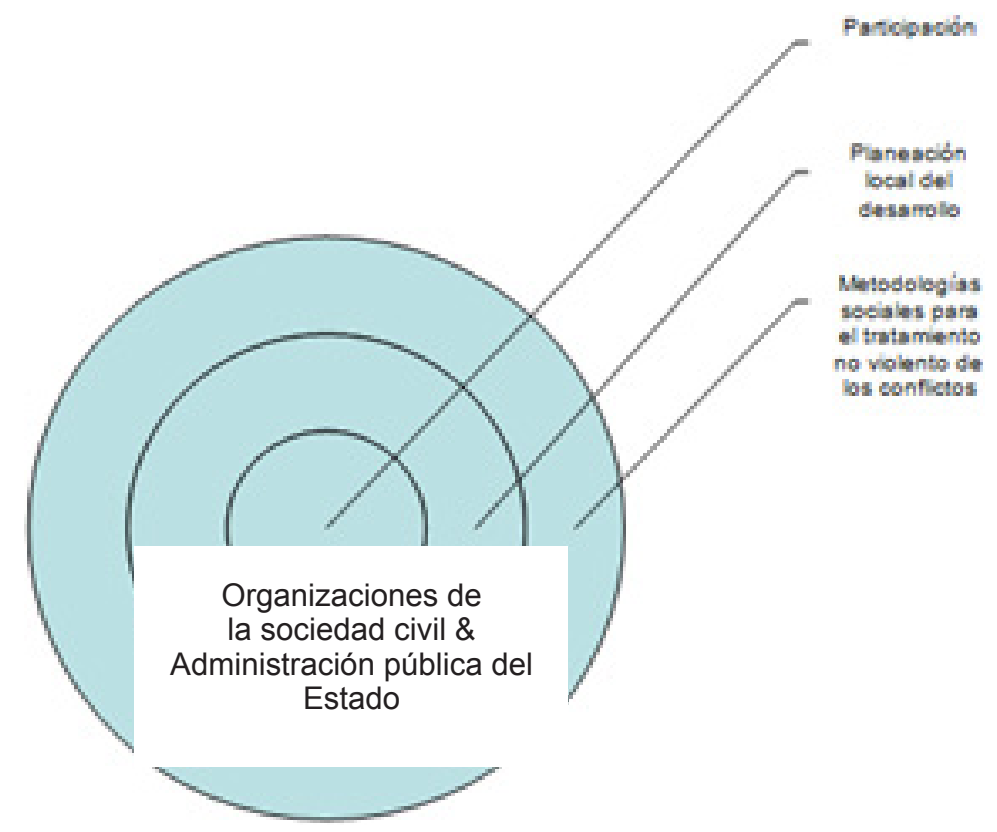

Figura 3. Panorama formativo de la convivencia ciudadana

Dar a entender entonces, a los ciudadanos y ciudadanas y especialmente a aquellos que cuentan con perfiles de liderazgo, responsabilidad social y trabajo comunitario, que la construcción democrática pasa por niveles de organización diversos y diferentes, permite entender que la canalización institucionalizada de demandas, la promoción de proyectos colectivos, contribuyen a la cualificación de la gestión pública y la vida ciudadana democrática.

El hilo conductor del proceso de formación debe asirse en la orientación de los procesos sociales en construcción permanente, como experiencias que posibilitan adquisiciones de capacidades, competencias y valores agregados en la triada ciudadano-comunidad, ciudadano-Estado y Estado-comunidad.

La construcción de nuevas sociabilidades pasa por el conocimiento de procesos de cualificación de liderazgo y participación social y cultural que faculten a los ciudadanos la incorporación de técnicas y procesos de convocatoria, sensibilización, organización y liderazgo social de cara a la construcción de procesos locales democráticos e incluyentes.

En este artículo se presenta una propuesta de construcción de ciudadanías en sociedades con altos niveles de conflictividad y crisis, como lo es el caso de la ciudad de Medellín, a través de una iniciativa pedagógica, esta iniciativa es entendida como un proceso que vincula aspectos politicos, sociales, culturales y legales en la perspectiva de formar nuevas ciudadanías (RAE, 2016). El artículo está distribuido de la siguiente manera: primero se presentan los referentes formativos a través de los materiales y métodos que sustentan la investigación. Como segundo se plantea la metodología y los resultados de la investigación con su respectiva discusión y como tercero se muestran las conclusiones y referencias bibliográficas. 


\section{Referentes formativos.}

La complejidad, densidad y diversidad cultural y política de sociedades que apuntan a la reconstrucción del tejido social, la recomposición del Estado y la recreación de culturas democráticas, deben orientar la pedagogía social y política en sentidos holísticos para formar ciudadanos activos con altos niveles de participación y organización.

Aspectos teóricos-prácticos de orden político-social democrático deben direccionar los sentidos de la ciudadanía, para tal efecto la formación de ciudadanías presupone la construcción-socialización de una cultura politica que transforme imaginarios sociales y modifique prácticas antidemocráticas de actuación social (Gary, 2002)

La pedagogización permanente en los escenarios cotidianos de propuestas de civilidad que articulen presupuestos y referentes incluyentes como la democracia, la pluralidad, la cultura de los Derechos humanos, las reflexiones de la sociedad civil, la escuela, la educación no formal pluridiversa, la opinión pública, los medios masivos de información, permiten construir referentes de vida politica-democrática como (Ver Figura 4):

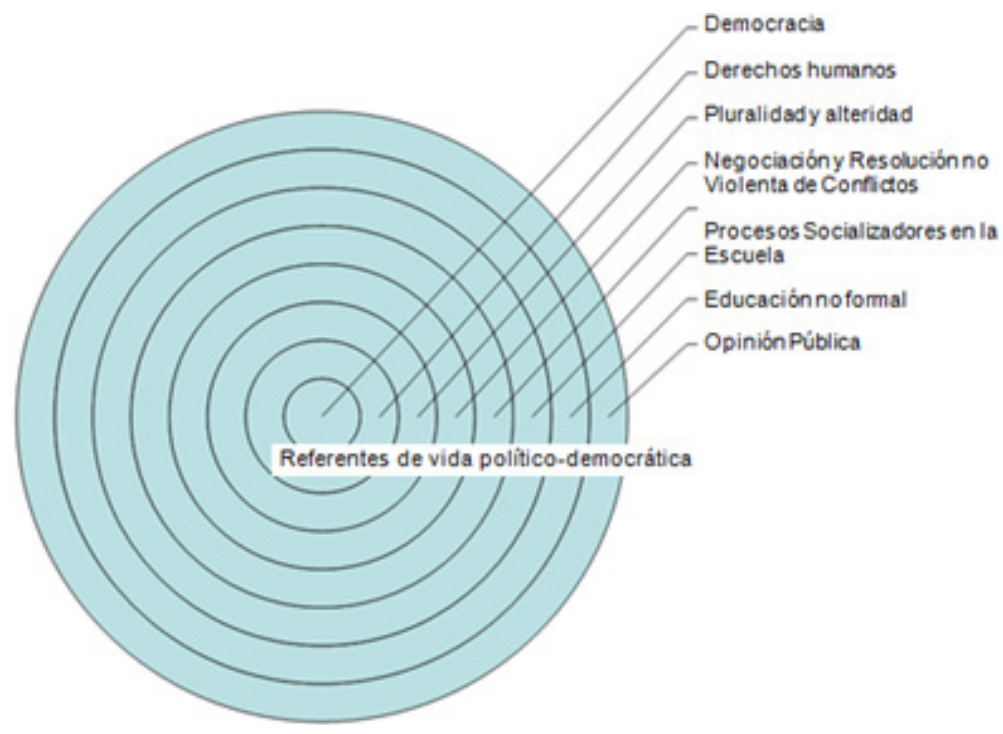

Figura 4. Escenarios de civilidad \& referentes de vida política-democrática

- Democracia: Como cultura abierta, participativa incluyente, que articule el sistema político a formas de vida y organización social macro y micro donde lo dialógico, argumentativo y consensuado constituye el norte de reflexión-actuación de la ciudadanía.

- Derechos humanos: como expresiones de organización societal y política civilizada y civilista que generan la cultura del respeto, dignificación y reconocimiento del ser humano, garantizando a éste desde un orden jurídico positivo, el desarrollo y la seguridad humana (Jelin \& Hershberg, 1996).

- Pluralidad y alteridad: Como condición polisémica de opiniones, concepciones, miradas, interpretaciones al igual que la diversidad de culturas, credos, partidos y organizaciones, razas, regiones y situaciones de género que permitan la construcción de un nosotros al 
servicio de fines altruistas de lo socio-político como construcción pública (Ramírez \& Parra Alfonso, 2009); (Glenn, 1999).

- Negociación y resolución no violenta de conflictos: Como la mediación, acuerdos y concertación, donde los ciudadanos y el Estado deben generar espacios y procedimientos civilizados tendientes a la convivencia ciudadana.

- Procesos socializadores en la escuela: Como instancia socializadora de saberes y conocimientos que trascienda la profesionalización, vinculada a proyectos políticos y socio-culturales de orden local, regional y nacional. (Torres, 1996)

- Educación no formal pluridiversa: Como espacio público de información, formación, deliberación, debate y decisiones que integre saberes formalizados con aquellos de la vida cotidiana y popular para incidir en los proyectos ciudadanos.

- Opinión pública: Como escenario cotidiano de producción de ideas, de crítica constructiva e inteligente, donde las libertades se materialicen como manifestación democrática, vinculando los medios de comunicación a expresiones sociales amplias e incluyentes, que permitan la circulación cierta de ideas, independiente de su procedencia y concepción.

Las estrategias deben posibilitar y materializar los procesos de acercamiento, de encuentro con la ciudad para fortalecer el proceso de construcción colectiva en la formulación, adopción e implementación de la propuesta pedagógica para la convivencia ciudadana y la formación de nuevas ciudadanías (Bonvillani, 2008) La pedagogía debe expresarse en estrategias que centren acciones de articulación de esfuerzos y recursos y que recoja propuestas para construir nuevas ciudadanias que contribuyan al fortalecimiento de la sociedad civil, con base en estos planteamientos se proponen tres estrategias (Ver Figura 5):

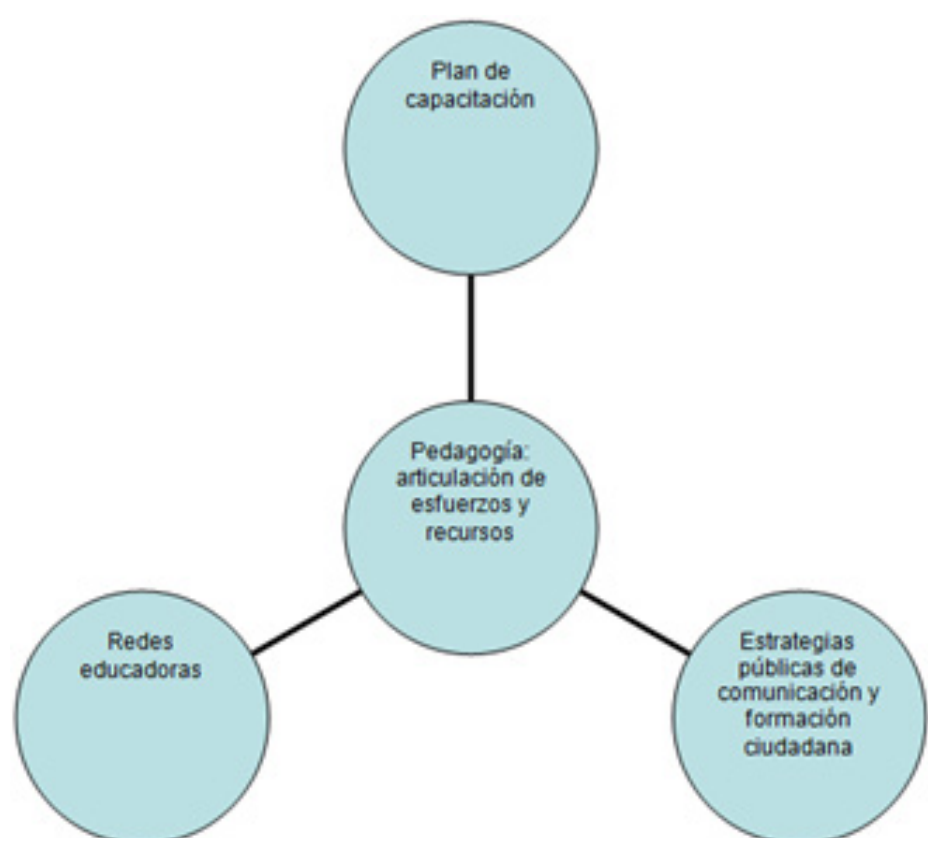

Figura 5. Lineamientos pedagógicos para la construcción de ciudadanía 
- Plan de capacitación. El proceso de capacitación para construir y fortalecer la implementación de la iniciativa de pedagogía política y social, debe ser estructurada, posicionada y reconocida como acción pedagógica de formación para abordar los retos que configuran la puesta en marcha de la formación ciudadana. Se propone el diseño colectivo de cursos formales e informales, donde se establezcan rutas académicas para abordar la composición de política en formación de ciudadanías democráticas, los planteamientos de la nueva gestión pública y social que se requiere y las demás resignificaciones conceptuales y operativas, necesarias para hacer posible lo formulado en la etapa inicial.

- Redes educadoras. Se configura como una estrategia pedagógica que permita la formación integral de ciudadanos y el redimensionamiento de las prácticas democráticas a nivel de los territorios, y de las formas organizativas que se materializan en la fase de implementación. Se debe contar con un programa de capacitación y planes de promoción de cultura ciudadana (campañas de comunicación social, entre otros), donde se fomenten los principios temáticos, filosóficos y operativos de la propuesta pedagógica.

- Estrategias públicas de comunicación y formación ciudadana. De cara a la pedagogización pública, se diseñan sus estrategias de divulgación, incluyendo elaboración de cartillas, material audiovisual que sea de amplia difusión en los medios masivos y alternativos de información y formación ciudadana.

\section{Procesos de formación.}

La formación ciudadana y los procesos de intervención comunitarios y societales, deben estar orientados sobre la base de la civilidad y la convivencia ciudadana, como escenario de las negociaciones y concertaciones locales (Ramírez E. , 2012). Por ello, los ciudadanos deben contar con espacios de reflexión que comprendan la importancia del diálogo y la concertación, como factor preponderante de la convivencia social.

La convivencia conlleva a formular los aspectos teóricos y prácticos básicos con referente amplio y democrático donde la Constitución (López, 2004) y la vigencia de la Cultura de la participación son, necesariamente, elementos importantes en la preparación para la vida en toda sociedad democrática y pluralista, más aún dentro de un proyecto de construcción y fortalecimiento de la ciudadanía.

Por ello, es importante dar a conocer a los ciudadanos y ciudadanas las opciones legales y legitimas de los escenarios, los mecanismos y la incidencia de la participación ciudadana en los procesos de planeación del desarrollo, en medio del fortalecimiento de la democracia y participación en el orden constitucional y legal.

Pedagogizar los aspectos sociales de la Constitución Política de Colombia (López, 2004) y la idea de integralidad y holismo de la convivencia como parte de las seguridades humanas, el bienestar con justicia social y la equidad inscrita en los modelos de desarrollo, permiten compartir la reflexión sobre la democracia, con análisis de las concepciones sociológicas y políticas implícitas en los derechos y deberes de participación y planeación social como impulsoras de la modernidad politica.

Planificar la gestión del desarrollo, exige posturas colectivas que reúnan deseos, necesidades y propuestas de la comunidad que conforma los escenarios locales, dentro de realidades diversas y complejas. 
Es imprescindible promover la planeación y participación como estrategias socializadoras que permitan visionar y trabajar por la ciudad que se quiere con seguridad y convivencia.
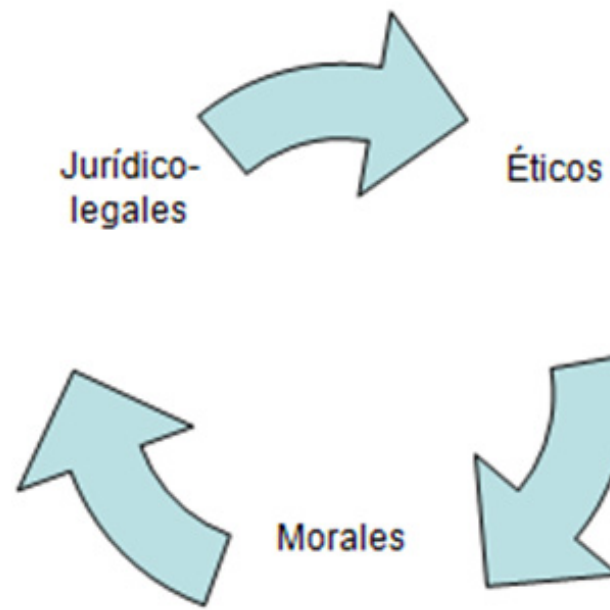

Morales

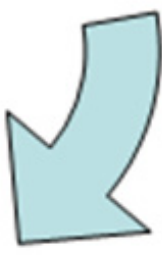

Figura 6. Indicadores básicos para el análisis de la propuesta

Para garantizar un discurso pertinente a las realidades societales que vivencian los ciudadanos en sus localidades, el proceso de formación tendrá indicadores básicos para el análisis, orientando la filosofia y propósito de esta propuesta pedagógica (Ver Figura 6):

- Los éticos. Que se refieren a las convicciones civilistas y que constituyen la parte fundamental de la estructura social, que se relaciona con los derechos y deberes de todo ciudadano, los cuales son interiorizados con reflexividad y conciencia en la acción ciudadana. (Savater, 1997)

- Los morales. En cuanto la conducta que moldea la acción humana porque se refiere a los valores que la persona comporta y expresa a través de la cultura ciudadana, que son los que determinan las relaciones sociales.

- Los jurídicos-legales. Que tocan con las reglas del juego formalizadas en el mundo institucional.

Se requiere la construcción de códigos de actuación donde la convicción y responsabilidad del ciudadano direccione su quehacer. Como complemento deben establecerse unas normas y valores que garanticen, además, el respeto por las distintas ideologías, credos religiosos, posiciones discursivas, aspectos de género y culturales (Díez, 2002).

Los valores fundamentales para la democracia son precisamente, la pluralidad, la convivencia y la participación entre la diversidad y heterogeneidad. Por lo tanto, es igualmente importante, construir como norma y como actitud la negociación, la concertación y el diálogo, como vías para el tratamiento no violento de los conflictos y la convivencia civilista.

Comprender que somos diferentes, que tenemos distintas visiones y posiciones frente a la vida; reconocer la necesidad que tenemos en "el otro" opciones para sobrevivir y realizarnos, lo que implica dialogar, negociar y concertar, es el primer paso para desencadenar la Cultura de la negociación y la concertación que contribuya a la armonía comunitaria y social. 
Los aportes concebidos en el proceso de capacitación, acompañamiento y estimulación ciudadana, pensado desde la participación, la planeación y los procesos locales como estrategias democráticas y promotoras del desarrollo comunitario y ciudadano, giran alrededor de priorizar sobre la información-formación integral y holística en las nuevas alternativas de gestión local para desarrollar procesos de construcción- transformación de liderazgos individuales y grupales, con carácter civilista y propositivo.

\section{Propuesta y aportes.}

\section{Dimensión práctica.}

Construir un capital político que acompañe a una iniciativa en formación ciudadana, requiere las garantías de participación activa de la misma en asuntos que tengan que ver con la planeación, organización y control de los planes de acción a seguir y la incidencia en los planes de inversión para esta implementación. Esto requiere una articulación para la formación, educación y proyección social, que se configura a través de las diferentes políticas, planes y programas que se impulsen en los distintos escenarios de la institucionalidad y de la sociedad civil. Los diversos actores sociales, culturales y políticos de la ciudad, que han venido interactuando en diferentes escenarios y con roles que responden a sus intencionalidades y enfoques de intervención respecto a la formación ciudadana requieren una orientación pedagógica que socialice y construya las visiones de las realidades territoriales particulares, las lecturas y vivencias del contexto.

\section{Aporte desde el nivel formativo.}

Se deben dinamizar procesos de formación que construyan conciencia de ciudad, de pluralidad, de encuentro colectivo para pensar salidas a las crisis de las comunas y barrios, la diversidad cultural y el reconocimiento de las dinámicas territoriales en un proyecto de sociedad en busca de la consolidación de la democracia, a través de la generación de niveles de bienestar social para la ciudadanía en general, un adecuado manejo del Estado y el ejercicio de derechos y libertades de todos los ciudadanos (Gary, 2002).

En este sentido la implementación de una política de formación ciudadana debe asegurarse de registrar procesos de formación, que se vienen dando en la ciudad, respecto a la formación de ciudadanías participativas como: los proyectos educativos institucionales de las escuelas y colegios, los programas de gobierno escolar, los programas de convivencia institucional de empresas y organizaciones de la ciudad, las escuelas de padres, las escuelas de liderazgos y de animación socio cultural de las organizaciones comunitarias; así se incluyen diferentes actores y escenarios para perfilar el programa de formación ciudadana.

Se contempla como estrategia, la articulación al proceso formativo formulado e implementado un el plan estratégico de ciudad, cuya intencionalidad debe ir dirigida a la construcción colectiva de ciudad a partir de la recuperación de la gobernabilidad, atravesada por la convivencia, el liderazgo regional, la organización social y el gobierno de la ciudad.

De igual manera promueve la percepción de calidad de vida con desarrollo social, económico, educativo, cultural y ambiental que se centre en la accesibilidad, la construcción de autoimagen territorial, la inversión y la disminución de vulnerabilidad social. 
Los lineamientos que orientan esta propuesta deben quedar registrados a lo largo de la política de formación ciudadana, en términos de los cambios deseados por la ciudad, para elevar las condiciones y calidades de vida e igualmente apostarle al proyecto de ciudad que se requiere.

Por lo tanto, los ejes orientadores del proceso de formación están dados en los siguientes aspectos:

- El fortalecimiento de la gestión política y social.

- La promoción de una noción de identidad sustentada en la red de Liderazgos sociales y comunitarios.

- La recuperación de los ámbitos y escenarios de ciudad: familia, escuela, barrio para las nuevas ciudadanías y culturas ciudadanas.

- El fomento de la cooperación público-privada.

- La proyección de la democracia participativa.

- El avance hacia una ética de lo público.

\section{Aporte desde el nivel educativo.}

El proceso educativo y formativo debe articularse a experiencias e instituciones educativas implementadas y desarrolladas en la ciudad de Medellín en torno a la convivencia pacífica. En este sentido, la iniciativa pedagógica debe inventariar la infraestructura física y de procesos con que la ciudad cuenta, debe respaldar la formulación de propuestas y las estrategias que pretende potenciar los nuevos lineamientos que sobre formación ciudadana se contemplan.

Partiendo de la concepción que sobre los territorios se ha sostenido, como construcción social, es necesario que la iniciativa pedagógica, conserve y fortalezca dicha intencionalidad, entendiendo por esta construcción el ejercicio práctico de inter-acción de actores, escenarios, enfoques e intereses.

Este debe comprender un proceso de planeación participativa entre las redes sociales (instituciones, actores, procesos y organizaciones territoriales) establecidas para pensar y perfilar los procesos educativos que deben conservarse, potencializarse o generarse. Igualmente, los contenidos que los integre y los procedimientos que deben llevarse a cabo para consolidar un proceso educativo para la vida ciudadana democrática con un carácter sostenido y progresivo.

Bajo esta perspectiva, la iniciativa pedagógica en formación ciudadana debe plegarse al proceso de ciudad educadora, por las implicaciones culturales que representa y que ha enfatizado en la concepción de la ciudad como el escenario educativo por excelencia, y en la ubicación de la educación como propósito colectivo para el desarrollo humano y social.

Es así como la iniciativa pedagógica desde el punto de vista de lo educativo, reafirma el interés de promover la responsabilidad de las instituciones, actores y escenarios; la modernización de la gestión y de los procesos para el fortalecimiento de la cultura política y el fomento de la democracia a través de la participación. 


\section{Aporte desde el nivel de proyección social.}

Desde el punto de vista de la proyección social, la iniciativa pedagógica debe garantizar la integralidad y la pertinencia de la convivencia ciudadana, en términos de contextos, territorios, particularidades de actores y escenarios. Es decir, que articule los diversos ámbitos de la práctica de los ciudadanos y ciudadanas que adquieren sentido en la vida cotidiana de las comunas y barrios de la ciudad de Medellín; por lo tanto, la pedagogía no será un ejercicio abstracto y vació de la política pública de convivencia.

El nivel de proyección social, orientado por la propuesta pedagógica debe partir de la experiencia y vivencias de los territorios y sus actores, para ser consecuentes con el carácter de complejidad social que atraviesa el análisis de la convivencia y la formación ciudadana. Esta dimensión de caracterización y expresión de las particularidades ciudadanas, deben complementarse con elaboraciones conceptuales, metodológicas y de contexto que operen apuestas prácticas para la convivencia, de modo que los procesos de aplicación puedan evidenciar transformaciones de la realidad en las dinámicas particulares de las complejidades sociales y en las dinámicas de relacionamiento de las redes pedagógicas.

Este componente de proyección social debe tener como punto de partida y de llegada momentos concretos, vivenciales, sistemáticos, creativos y colectivos de los escenarios y actores respecto a la implementación de los programas de un plan de gestión, con un alto contenido de trabajo conceptual-pedagógico y didáctico para la construcción participativa de actos reales y medibles.

\section{Conclusiones.}

Acompañar a la ciudad de Medellín, en un proceso de construcción de cultura ciudadana exige una búsqueda colectiva en dimensiones particulares y sociales inscritas en principios democráticos, incluyentes y de desarrollo humano y social, donde se permita un ordenamiento urbano para la equidad y la integralidad de la vida ciudadana. Por lo tanto un proceso de pedagogía para la formación y la convivencia ciudadana requiere una aproximación evolutiva de formación, educación y de proyección social, que permita construir el porqué y el para qué de las normas y lineamientos, el qué de la conciencia social y el cómo y el con qué de las intencionalidades reales de la propuesta, para que así la multiplicidad de fuentes generadoras de inseguridades y violencias también comprendan una multiplicidad de procesos para las estrategias, metodologias y herramientas de respuesta.

Como trabajo futuro de esta investigación se propone la inclusión de herramientas TIC para la formación de todos los agentes que se incluyen en los diferentes escenarios de la iniciativa. Específicamente, una plataforma que facilite los procesos de enseñanza y de aprendizaje a través de la red internet, en donde se pueda acceder a los contenidos y actividades educativas sin importar el lugar y el tiempo. 


\section{Referencias bibliográficas.}

Álvarez, S., Dagnino, E., \& Escobar, A. (1998). Cultures of politics, politics of cultures: re-visioning Latin American social movements. Boulder, CO: Westview Press.

Bonvillani, A. (2008). Construcción de ciudadanía "desde abajo": Posibilidades y límites en la experiencia de un grupo de jovenes pobres. Pensares, 459-478.

Botia, A. B. (1998). Educar en valores: una educación de la ciudadanía. Sevilla: Junta de Andalucía.

Castillo Castillo, M. L. (julio-diciembre de 2014). el rol de la democracia deliberativa y su ejercicio. El Ágora USB, 14(2), 473-486. Obtenido de http://revistas.usb.edu.co/index.php/Agora/ article/view/25/41

Díez, R. (2002). Aprender para el futuro. Educación a favor de la dignidad humana. Madrid: Santillana.

Gary, L. (2002). Ciudadanía, lo público, democracia. Bogotá: Litocinco.

Glenn, E. (1999). The social construction and institutionalization of gender and race. Revisioning gender, 3-43.

Jelin, E., \& Hershberg, E. (1996). Constructing democracy: human rights, citizenship, and society in Latin America. Westview Pr.

López, J. O. (2004). Constitución politica de Colombia. Bogotá: Plaza y Janes Editores Colombia S. A.

Muñoz Gaviria, D. (2011). El compromiso social de las facultades de educación: reflexiones pedagógicas en torno a la educación y la crisis de la modernidad. El Ágora USB, 11(1), 125152. Obtenido de http://revistas.usb.edu.co/index.php/Agora/article/view/390/149

RAE. (2016). Real Academia Española. Obtenido de www.rae.es.

Ramírez, D., \& Parra Alfonso, G. (2009). La ética del cuidado. Hacia la construcción de nuevas ciudadanías. Psicología desde el Caribe, 183-213.

Ramírez, E. (2012). Formación para la democracia y la convivencia ciudadana. Medellin: Universidad Nacional de Colombia.

Reina, V. G. (1998). El aprendizaje cívico. Barcelona: Ariel.

Savater, F. (1997). El valor de educar. Barcelona: Ariel.

Torres, C. A. (1996). Las secretas aventuras del orden: Estado y Educación. Miño y Dávila Editores.

Vargas, V. (2001). Ciudadanias globales y sociedades civiles globales.Pistas para el analisis. Obtenido de www.agenciatoro.com.br: http://www.agenciatoro.com.br/fjdlac/conteudos/ artigos/art02.pdf 